\title{
Recalling and Forgetting Dreams: Theta and Alpha Oscillations during Sleep Predict Subsequent Dream Recall
}

\author{
Cristina Marzano, ${ }^{1}$ Michele Ferrara, ${ }^{2}$ Federica Mauro, ${ }^{1}$ Fabio Moroni, ${ }^{1,3}$ Maurizio Gorgoni, ${ }^{1}$ Daniela Tempesta, ${ }^{2}$ \\ Carlo Cipolli, ${ }^{3}$ and Luigi De Gennaro ${ }^{1,4}$ \\ ${ }^{1}$ Department of Psychology, University of Rome “Sapienza," 00185 Rome, Italy, 2Department of Health Sciences, University of L'Aquila, 67010 L'Aquila, \\ Italy, ${ }^{3}$ Department of Psychology, University of Bologna, 40127 Bologna, Italy, and ${ }^{4}$ Associazione Fatebenefratelli per la Ricerca, Department of \\ Neuroscience, Hosp. Fatebenefratelli, Isola Tiberina, 00186 Rome, Italy
}

Under the assumption that dream recall is a peculiar form of declarative memory, we have hypothesized that (1) the encoding of dream contents during sleep should share some electrophysiological mechanisms with the encoding of episodic memories of the awake brain and (2) recalling a dream(s) after awakening from non-rapid eye movement (NREM) and rapid eye movement (REM) sleep should be associated with different brain oscillations. Here, we report that cortical brain oscillations of human sleep are predictive of successful dream recall. In particular, after morning awakening from REM sleep, a higher frontal 5-7 Hz (theta) activity was associated with successful dream recall. This finding mirrors the increase in frontal theta activity during successful encoding of episodic memories in wakefulness. Moreover, in keeping with the different EEG background, a different predictive relationship was found after awakening from stage 2 NREM sleep. Specifically, a lower $8-12 \mathrm{~Hz}$ (alpha) oscillatory activity of the right temporal area was associated with a successful dream recall. These findings provide the first evidence of univocal cortical electroencephalographic correlates of dream recall, suggesting that the neurophysiological mechanisms underlying the encoding and recall of episodic memories may remain the same across different states of consciousness.

\section{Introduction}

Although studied extensively in the last 50 years, the brain mechanisms underlying the elaboration of dreaming and its encoding in memory during sleep are as yet primarily unknown, as a consequence of two limitations. First, since dreams are not directly accessible, the study of dreaming has been restricted to the analysis of mental activity after spontaneous or provoked awakening. Second, the study of the brain mechanisms involved in dreaming has usually been restricted to rapid eye movement (REM) sleep, on the basis of the implicit assumption that dreaming is strictly dependent on its specific physiology. However, dream recall is also obtained after awakening from all non-rapid eye movement (NREM) stages, with some difference in the frequency and content characteristics (Nielsen, 2000; Fagioli, 2002).

In the present investigation, we renounced to study dream reports and their characteristics and focused on the EEG oscillations associated with the presence/absence of dream recall after awakening. The basic assumption is that dream recall regards such peculiar form of episodic information as dream content

Received Jan. 25, 2011; revised March 2, 2011; accepted March 13, 2011

Author contributions: C.M., M.F., C.C., and L.D.G. designed research; C.M., F. Mauro, F. Moroni, M.G., and D.T. performed research; L.D.G. analyzed data; and C.M. and L.D.G. wrote the paper.

This work was supported by grants from the Compagnia di San Paolo, Programma Neuroscienze 2008/09 (3889 SD/sd, 2008.1300 to L.D.G.) and Fondazione CARISBO (2010.0139 to C.C. and F. Moroni). We thank Clayton Dickson and Jeremy Caplan for providing the libraries for the BOSC detection method.

Correspondence should be addressed to Luigi De Gennaro, Department of Psychology, Section of Neuroscience, University of Rome "Sapienza," Via dei Marsi, 78, 00185 Rome, Italy. E-mail: luigi.degennaro@uniroma1.it.

DOI:10.1523/JNEUROSCI.0412-11.2011

Copyright $\odot 2011$ the authors $\quad 0270-6474 / 11 / 316674-10 \$ 15.00 / 0$
(Fosse et al., 2003), which is encoded in declarative memory during sleep. Two main hypotheses directly descend from this assumption: (1) the encoding of dream content during sleep should share some electrophysiological mechanisms with the successful encoding of episodic information during wakefulness; and (2) resulting from the intrinsic electrophysiology of REM and NREM sleep, dream recall after awakening from these sleep states should be associated with different brain oscillations.

These hypotheses rely on a large body of experimental evidence. In humans engaged in a learning task, a successful memory formation is predicted by a tight coordination of spike timing with local theta oscillations (Rutishauser et al., 2010). Moreover, evoked potentials and hemodynamic measures of human brain activity differentiate the encoding of items to be later recalled from that of items not later recalled (Paller and Wagner, 2002). Scalp recordings have shown that low-frequency brain oscillations increase during encoding of episodic memories to be later recalled (Klimesch et al., 1996; Weiss and Rappelsberger, 2000; Osipova et al., 2006). Intracranial recordings have confirmed that an increase in frontal theta oscillations during the encoding phase predicts subsequent recall (Sederberg et al., 2003) and that theta oscillations in wakefulness mediate interactions between prefrontal cortex and medial temporal lobe in memory encoding (Anderson et al., 2010).

Keeping in mind the main EEG difference between REM and NREM sleep, we attempted to identify which specific electroencephalographic activity within localized cortical areas is associated with dream recall after awakening from different sleep stages. Concerning REM sleep, we expected that a higher frontal 
theta activity should be associated with the awakenings followed by successful dream recall compared with those with recall failure. Indeed, a recent investigation has shown that the extent of postsleep facilitation for emotional memory is correlated with prefrontal theta oscillations only during REM sleep (Nishida et al., 2009).

According to previous observations (Esposito et al., 2004), we expected that a successful recall after awakening from NREM stage 2 should be associated with a lower alpha power compared with awakening with no dream recall, larger in corresponding temporo-parietal areas.

Here, we report that a higher frontal theta activity during REM sleep and a lower alpha activity of the right temporal region during NREM sleep actually predict successful dream recall after awakening. Both these findings are further strengthened by the significant correlations between the number of recalled dreams and these stage-specific brain oscillations during sleep.

\section{Materials and Methods}

Subjects

Data for this analysis were obtained by selecting an ad hoc sample of 65 university students [ 35 males $(\mathrm{M})$ and 30 females (F); mean age, $23.6 \pm$ 2.1 years] from the database of our laboratory. Subjects were selected from those who participated to distinct protocols (De Gennaro et al., 2008, 2010; C. Marzano, M. Ferrara, F. Moroni, and L. De Gennaro, unpublished observations), on the basis of the following study-specific criteria: (1) polysomnographic (PSG) recordings of 2 consecutive nights (for adaptation and baseline, respectively) of undisturbed sleep; (2) the collection of a sleep and dream diary (see below) after morning awakening in the second night; and (3) the absence of stage shifts in the last $5 \mathrm{~min}$ of sleep preceding the provoked awakening at the end of the second night.

Additionally, the subjects had to meet the following general criteria: normal sleep duration and schedule; no daytime nap habits; no excessive daytime sleepiness; no other sleep, medical, or psychiatric disorder (as assessed by a 1 week sleep log and by a clinical interview); a habitual sleep time between 12:00 A.M. and 8:00 A.M. plus or minus $1 \mathrm{~h}$; and an average sleep duration of $7.5 \pm 1$ h hours per night.

In such a way, we considered 35 ( $16 \mathrm{M}$ and $19 \mathrm{~F})$ subjects awakened from stage 2 sleep and 30 (19 M and $11 \mathrm{~F}$ ) from REM sleep, also taking into consideration the need to have comparable sizes for the groups recalling and nonrecalling dreams after either REM or stage 2 sleep.

The protocol of the present study was approved by the local Institutional Ethics Committee and was conducted in accordance with the Declaration of Helsinki. All subjects had previously given their written informed consent for the collection and treatment of data.

\section{Procedure}

For each subject, a polysomnogram was recorded in a sound-proof, temperature-controlled room after an adaptation night. Hence, the recordings were obtained during second nights in the sleep laboratory, and any procedural aspect was completely identical to the first adaptation night. The subjects' sleep was undisturbed, started at 12:00 A.M., and ended after $\sim 7.5 \mathrm{~h}$ of accumulated sleep. Awakenings were scheduled from stage 2 sleep $(n=35)$ or from REM sleep $(n=30)$ after at least $5 \mathrm{~min}$ without stage shifts (by calling out his/her name and entering in the sleep room). The decision to wake up the subject was taken by the sleep researcher who examined PSG recording online, and the absence of stage shifts over the last 5 min of sleep was then confirmed by an independent expert, who off-line scored PSG recording.

Just after morning awakening, subjects were requested to fill out a sleep and dream diary (De Gennaro et al., 2010), to collect subjective estimates of sleep characteristics (sleep onset latency, number of awakenings, total sleep time, sleep quality) and of dream recall, namely the feeling of having or having not dreamt (as a qualitative estimate) and the number of recalled dreams of the preceding night (as a quantitative
Table 1. Means and SEs of the PSG variables of the REC and NREC groups awakened from REM sleep

\begin{tabular}{|c|c|c|c|c|c|c|}
\hline \multirow[b]{2}{*}{ Variables } & \multicolumn{2}{|l|}{ REC } & \multicolumn{2}{|l|}{ NREC } & \multirow[b]{2}{*}{$F_{(1,28)}$} & \multirow[b]{2}{*}{$p$} \\
\hline & Mean & SE & Mean & SE & & \\
\hline Stage 1 latency (min) & 7.32 & 1.55 & 9.47 & 2.35 & 0.61 & 0.44 \\
\hline Stage 2 latency (min) & 11.11 & 2.32 & 13.27 & 2.37 & 0.34 & 0.56 \\
\hline REM latency (min) & 84.78 & 6.87 & 86.00 & 11.36 & 0.009 & 0.92 \\
\hline Stage $1(\%)$ & 6.28 & 0.68 & 6.64 & 1.18 & 0.08 & 0.78 \\
\hline Stage $2(\%)$ & 60.00 & 1.39 & 59.26 & 1.54 & 0.11 & 0.74 \\
\hline SWS (\%) & 10.54 & 1.62 & 8.16 & 1.45 & 0.89 & 0.35 \\
\hline REM (\%) & 23.17 & 0.88 & 25.94 & 1.70 & 2.58 & 0.12 \\
\hline WASO (min) & 32.01 & 8.42 & 23.56 & 4.10 & 0.47 & 0.50 \\
\hline TST (min) & 443.30 & 9.32 & 435.27 & 8.79 & 0.30 & 0.59 \\
\hline SEI \% (TST/TBT) & 91.98 & 1.63 & 92.87 & 1.08 & 0.13 & 0.72 \\
\hline
\end{tabular}

The results of the one-way ANOVA are also reported.

Table 2. Means and SEs of the PSG variables of the REC and NREC groups awakened from stage 2

\begin{tabular}{|c|c|c|c|c|c|c|}
\hline \multirow[b]{2}{*}{ Variables } & \multicolumn{2}{|l|}{ REC } & \multicolumn{2}{|l|}{ NREC } & \multirow[b]{2}{*}{$F_{(1,33)}$} & \multirow[b]{2}{*}{$p$} \\
\hline & Mean & SE & Mean & SE & & \\
\hline Stage 1 latency (min) & 8.89 & 2.08 & 9.51 & 2.94 & 0.03 & 0.86 \\
\hline Stage 2 latency ( $\mathrm{min}$ ) & 12.93 & 2.44 & 16.02 & 4.50 & 0.41 & 0.52 \\
\hline REM latency (min) & 91.64 & 6.09 & 87.09 & 9.34 & 0.18 & 0.67 \\
\hline Stage $1(\%)$ & 11.92 & 5.26 & 7.17 & 0.88 & 0.60 & 0.44 \\
\hline Stage $2(\%)$ & 61.77 & 1.62 & 58.60 & 1.82 & 1.69 & 0.20 \\
\hline SWS (\%) & 9.50 & 1.49 & 10.57 & 1.67 & 0.23 & 0.64 \\
\hline REM (\%) & 21.81 & 1.07 & 23.66 & 1.27 & 1.24 & 0.27 \\
\hline WASO (min) & 31.51 & 4.74 & 40.44 & 9.29 & 0.84 & 0.36 \\
\hline TST (min) & 441.72 & 6.20 & 437.00 & 14.43 & 0.07 & 0.80 \\
\hline SEI \% (TST/TBT) & 88.94 & 1.98 & 88.43 & 2.41 & 0.03 & 0.87 \\
\hline
\end{tabular}

The results of the one-way ANOVA are also reported.

estimate). To this aim, subjects had been preliminarily (i.e., at the end of the adaptation night) instructed to consider any distinct mental activity occurring during sleep with one or more perceptually vivid contents as a dream.

Of 35 subjects awakened from stage 2 sleep, 20 were successful in dream recall [recallers (REC); mean number of recalled dreams, 1.65; SE, 0.17 ] and 15 were not successful [nonrecallers (NREC)]. Of 30 subjects awakened from REM sleep, 20 were dream REC (mean number of recalled dreams, 1.32; SE, 0.13) and 10 were NREC. These recall rates were slightly different from the normative values of dream recall after morning awakening from REM and stage 2 sleep (Nielsen, 2000), because of the constrains of the current experimental design (mainly, the need to have comparable sample sizes and the absence of stage shifts over the last $5 \mathrm{~min}$ of sleep). Actually, the dream recall rates in the original database (De Gennaro et al., 2008; 2010; Marzano, Ferrara, Moroni, and De Gennaro, unpublished observations) were $69 \%$ for the REM awakenings and $51 \%$ for the stage 2 awakenings.

\section{PSG recordings}

A VEGA 24 polygraph (Esaote Biomedica) was used for polygraphic recordings. EEG signals were analogically high-pass filtered with a time constant of $0.3 \mathrm{~s}$ and low-pass filtered at $30 \mathrm{~Hz}$. The 19 unipolar EEG derivations of the international 10-20 system (Fp1, Fp2, F7, F8, F3, F4, $\mathrm{Fz}, \mathrm{C} 3, \mathrm{C} 4, \mathrm{Cz}, \mathrm{P} 3, \mathrm{P} 4, \mathrm{Pz}, \mathrm{T} 3, \mathrm{~T} 4, \mathrm{~T} 5, \mathrm{~T} 6, \mathrm{O} 1, \mathrm{O} 2)$ were recorded from scalp electrodes with averaged mastoid reference.

The submental EMG was recorded with a time constant of $0.03 \mathrm{~s}$. Bipolar horizontal eye movements were recorded with a time constant of $1 \mathrm{~s}$. The bipolar horizontal electrooculogram (EOG) was recorded from electrodes placed about $1 \mathrm{~cm}$ from the medial and lateral canthi of the dominant eye. Impedance of these electrodes was kept below $5 \mathrm{k} \Omega$.

\section{Data analysis}

Polysomnography. Sleep stages were visually scored according to the standard criteria (Rechtschaffen and Kales, 1968), and slow-wave sleep 
A

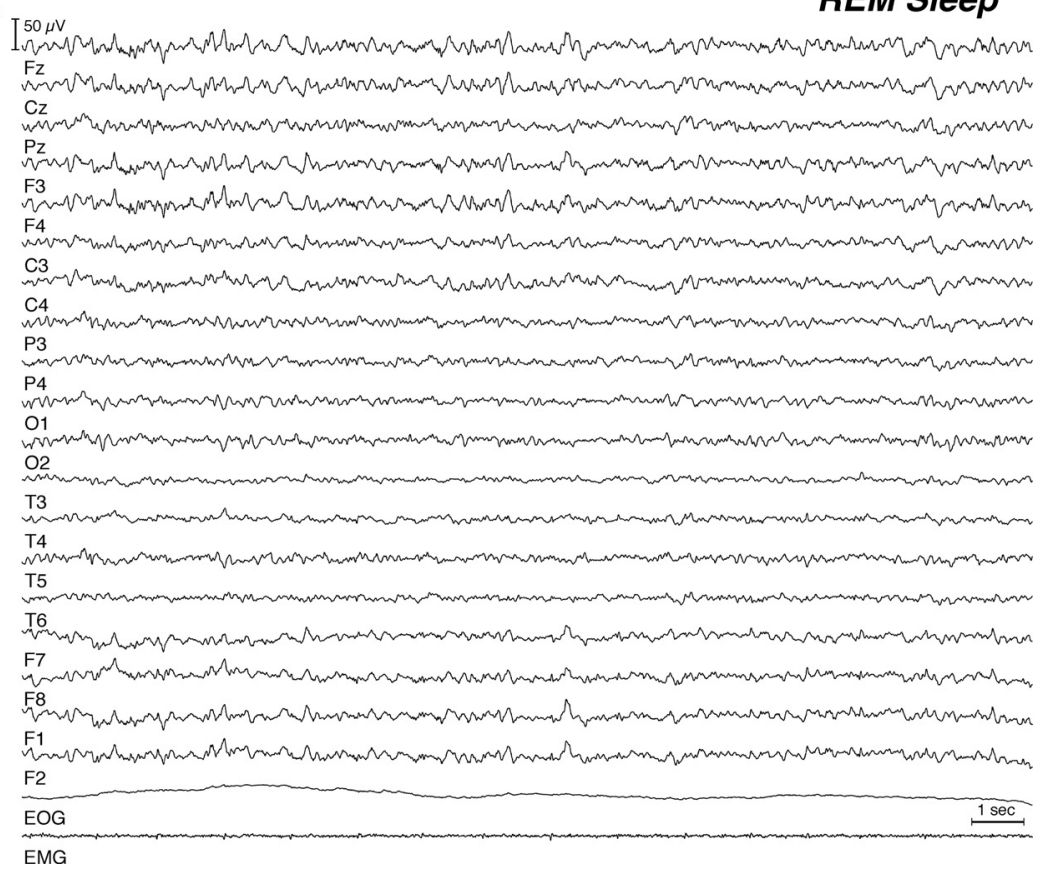

B

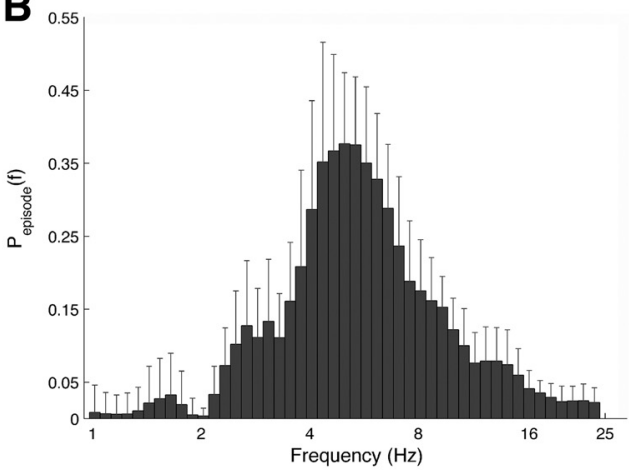

Figure 1. REM sleep. $A$, One 20 s segment of the PSG recording of tonic REM sleep preceding morning awakening. $B$, The mean proportion of time $\left[P_{\text {episode }}(f)\right]$ of the EEG segment shown in $A$, where oscillations were detected at each frequency in the $0.25-25.00 \mathrm{~Hz}$ frequency range. The detection of oscillations has been made by the BOSC detection method (see Materials and Methods) on the $19 \mathrm{EEG}$ electrodes. Error bars denote interlocation variability. Units of frequency are in hertz and are plotted in five logarithmically spaced frequency values.

A

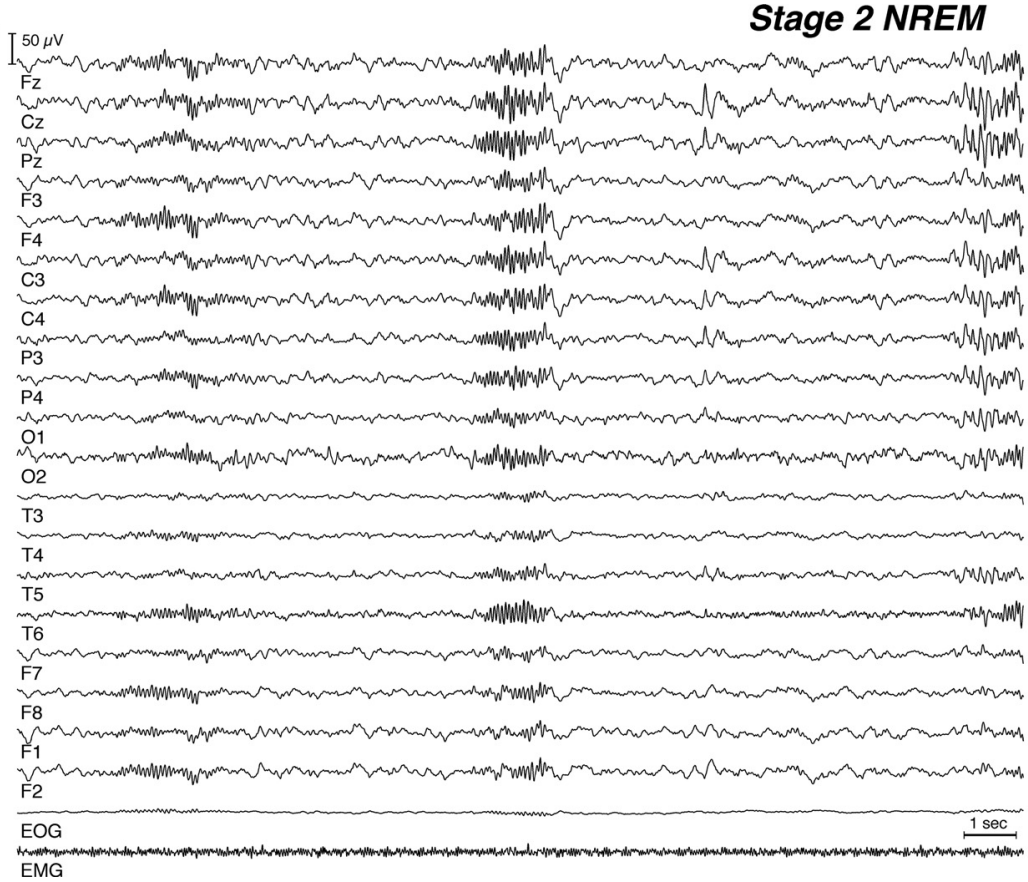

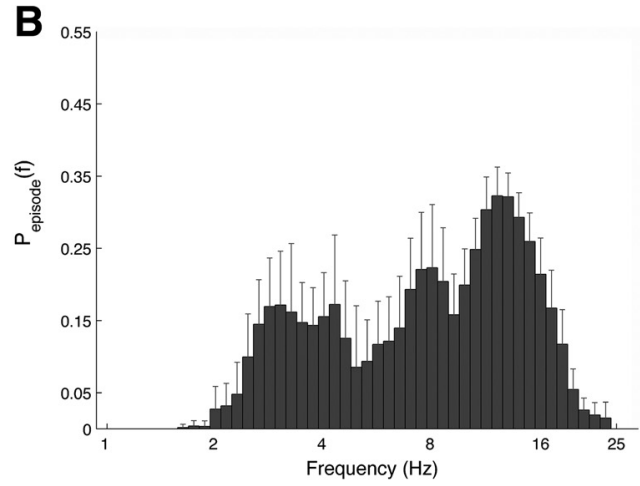

Figure 2. Stage 2 sleep. $A, 0$ ne 20 s segment of the PSG recording of stage 2 sleep preceding morning awakening. $B$, The detection of oscillations in the segment of stage 2 sleep by the BOSC method, as in Figure 1.

(SWS) scoring strictly followed the $>75 \mu \mathrm{V}$ amplitude criterion. The following sleep parameters were considered as dependent variables: (1) stage 1 latency; (2) stage 2 latency; (3) REM latency; (4) total sleep time (TST), defined as the sum of time spent in stage 1, stage 2, SWS, and REM; (5) sleep efficiency index (SEI $=$ TST/total bed time $(\mathrm{TBT}) \times$ $100)$; (6) percentage of each sleep stage (time spent in a sleep stage/ TST); and (7) wakefulness after sleep onset (WASO), expressed as the intrasleep time (minutes) spent awake.
To ascertain whether REC and NREC subjects differed with respect to one or more PSG measures, we performed one-way ANOVAs separately for the two groups with awakening from REM or stage 2 NREM sleep.

Data processing and statistics. The polygraphic signals (19 EEG channels, an EMG, and an EOG) of the 5 min of sleep preceding the morning awakening were analog-to-digital converted on-line with a sampling rate of $128 \mathrm{~Hz}$ and stored on the disk of a personal computer. Artifacts were 
excluded off-line on an $8 \mathrm{~s}$ basis by visual inspection; with regard to REM sleep, only tonic periods were included in the analyses, to avoid artifacts from rapid eye movements on EEG power. Power spectra of the 19 derivations were computed by a fast Fourier transform (FFT) routine for consecutive $4 \mathrm{~s}$ epochs, resulting in a frequency resolution of $0.25 \mathrm{~Hz}$. Values above $25 \mathrm{~Hz}$ were not used in the analysis.

Power EEG data at this frequency resolution were first averaged over all electrodes to provide a synthetic picture of EEG spectra as a function of the different conditions (REC and NREC awakened from REM sleep and from stage 2 NREM sleep).

Then, adjacent $0.25 \mathrm{~Hz}$ bins $(0.50-25 \mathrm{~Hz})$ were collapsed, reducing power EEG data to the following bands: delta $(0.50-4.75 \mathrm{~Hz})$, theta $(5.00-7.75 \mathrm{~Hz})$, alpha $(8.00-11.75 \mathrm{~Hz})$, sigma $(12.00-15.75 \mathrm{~Hz})$, and beta $(16.00-24.75 \mathrm{~Hz})$. The EEG power values for each frequency band were considered as dependent measures for the statistical analyses. For graphical purposes, these values were normalized by total power of the recording, color coded, plotted at the corresponding position on the planar projection of the scalp surface, and interpolated (biharmonic spline) between electrodes.

EEG power maps were computed separately for REM and stage 2 sleep, and statistical comparisons (unpaired $t$ tests) were performed on logtransformed values between REC and NREC groups, separately for the two sleep stages. To adjust $\alpha$ value for multiple comparisons, the Bonferroni correction was applied taking into account the mean correlation between the dependent variables (Perneger, 1998; Sankoh et al., 1997). According to this procedure, we calculated the mean correlation between the dependent variables in REM $(r=0.58)$ and NREM $(r=0.47)$ sleep. Considering this mean correlation and the number of statistical comparisons ( $95 t$ tests), $\alpha$ value was adjusted to 0.007 ( $t \geq 2.60$ ) for REM and to $0.004(t \geq 2.78)$ for NREM statistical comparisons.

Aimed to confirm the results of the $t$ maps, the existence of significant changes of theta activity in the comparisons between REC and NREC subjects during REM sleep localized over the frontal region also has been evaluated by a $2 \times 5 \times 19$ mixed design, group (REC vs NREC) $\times$ band (delta, theta, alpha, sigma, beta) $\times$ scalp location (the 19 derivations of the 10-20 international system) ANOVA on log-transformed EEG power values. Separate ANOVAs have been performed for REM and stage 2 sleep.

Then, to strengthen the results of the FFT analysis, we also applied an oscillation detection method to the EEG signals. Indeed, the presence of a spectral peak resulting from a Fourier transform of the EEG signal does not necessarily imply an underlying oscillatory activity at that frequency, given that nonoscillatory and transient signals can produce power changes at specific frequencies. For this reason, we also applied the better oscillation (BOSC) detection method to the EEG signals. The BOSC is a method, introduced by Caplan et al. (2001) and recently revised by Whitten et al. (2010), that is aimed to detect oscillatory activity within a signal containing a nonrhythmic portion. This method detects oscillatory activity in EEG signals by taking into account the functional form of a "background," nonrhythmic portion of the signal and revealing segments of the recording that deviate significantly from the spectral characteristics of the background.

The analysis was performed separately at each frequency of interest (in the $0.50-25 \mathrm{~Hz}$ range) and each electrode. For a given frequency, an oscillatory episode was defined as an epoch longer than a duration threshold, $D_{\mathrm{T}}$ (set to three cycles in our analysis) during which wavelet power at frequency exceeded a power threshold $\left(p_{\mathrm{T}}\right)$. This $P_{\mathrm{T}}$ was chosen as follows in the 5 min segments. (1) The EEG was wavelet transformed (Morlet wavelet, window of 6 cycles) at 47 logarithmically spaced frequencies in the range $0.50-25 \mathrm{~Hz}$. The average of the log-transform of these wavelet values yielded the wavelet power spectrum. (2) The background noise spectrum assumed the form $\operatorname{Power}(f)=A f^{-\alpha}$. The estimate of this background has been obtained by fitting the observed spectrum (at each electrode) with a linear regression in log-log units. The background at $f^{*}$ has been estimated on the mean of its corresponding $\chi^{2}(2)$ probability distribution function. The power threshold $\left(p_{\mathrm{T}}\right)$ was set to the 95th percentile of the theoretical probability distribution. The proportion of time in which significant oscillations were detected within

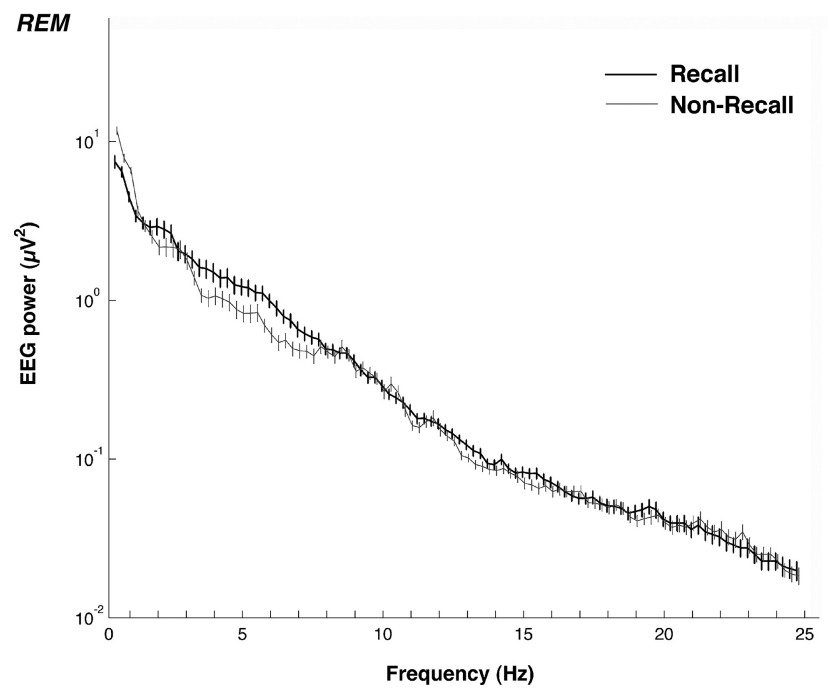

Figure 3. EEG power spectra (mean over all 19 scalp locations, obtained by collapsing EEG power across all the derivations) of EEG activity during REM sleep in the REC (black line) and NREC (gray line) groups. Mean absolute values (expressed in logarithmic scale) are plotted in the frequency range from 0.50 to $24.75 \mathrm{~Hz}$ for $0.25 \mathrm{~Hz}$ bins. SEs refer to interlocation variability.

a 5 min sleep was termed $P_{\text {episode }}$ (Caplan and Glaholt, 2007; Caplan et al., 2001, 2003; van Vugt et al., 2007).

The BOSC is a robust detection method that has been successfully used to identify and quantify theta oscillations in the human neocortex (Caplan and Glaholt, 2007; Caplan et al., 2001, 2003) and hippocampus (Ekstrom et al., 2005), which were correlated with memory encoding and retrieval. Here, the $P_{\text {episode }}$ measure provided by the BOSC method at each frequency $\left[P_{\text {episode }}(f)\right]$ was used to detect the oscillatory activity within those electrodes and EEG bands that had previously found to predict the presence of dream recall after awakening from REM and stage 2 on the basis of the FFT analysis.

As a final control, we checked whether the results on the final $5 \mathrm{~min}$ of sleep represent a pattern fairly stable over the night. In fact, our choice of studying EEG activity just preceding final morning awakening was mainly attributable to methodological reasons: (1) to more easily assume a quasi-stationarity of EEG signals; (2) to be closer to the time in which dream was retrieved; (3) to avoid the potential confound of the effects of homeostatic and ultradian factors on EEG changes across a night sleep.

Nevertheless, as a consequence of this methodological choice, it may remain unclear whether any association between sleep EEG oscillations and dream recall applies only to a short interval preceding awakening or whether it represents an interindividual pattern that may be detected throughout the night. To specifically address this question, 5 min EEG segments were selected around the midpoint of three episodes of REM sleep and stage 2 during the course of the night. To this aim, we considered the second, third, and fourth sleep cycles (i.e., NREM-REM cycles). The first cycle was discarded because its short duration and the stage shifts of the first REM period did not allow us to fulfill the criterion of a continuous 5 min segment in the middle of the sleep cycle. The fourth sleep episode was also not considered in the cases in which the $5 \mathrm{~min}$ central EEG segment would have been partly overlapped with the final 5 min interval before the final awakening. Therefore, the entire subject sample was included in the analyses on the second and the third sleep cycles, whereas 22 subjects ( 15 REC and 7 NREC) were considered for the analyses on the fourth REM sleep episode and 33 subjects (19 REC and 14 NREC) were considered for those on the fourth stage 2 episode. Sleep cycles were defined according to standard criteria (Feinberg and Floyd, 1979).

EEG power maps have been computed separately for REM and stage 2 sleep, and statistical comparisons (unpaired $t$ tests) have been performed on log-transformed values between REC and NREC groups, separately for the two sleep stages and for the three sleep cycles. Considering the mean correlation between dependent variables in REM $(r=0.30)$ and 
NREM sleep $(r=0.36)$, and the number of statistical comparisons (57 $t$ tests), $\alpha$ value was adjusted to 0.003 ( $t \geq 3.24$ for second and third cycles; $t \geq 3.38$ for fourth cycle) for REM sleep and to 0.004 ( $t \geq 3.12$ for second and third cycles; $t \geq 3.13$ for fourth cycle) for stage 2 NREM sleep statistical comparisons.

\section{Results}

PSG measures do not differ in REC and NREC groups

Tables 1 and 2, respectively, report the results of the univariate ANOVAs comparing the values of PSG measures in the REC and NREC groups, awakened from REM and stage 2 sleep. Comparisons do not show significant changes for any considered measure. This means that there were no substantial differences in the sleep architecture of the subjects grouped as a function of sleep stage at awakening (REM/stage 2) and dream recall (success/failure).

\section{Sleep EEG topography before} awakening discriminates between recalling and nonrecalling dreams after awakening

A representative segment of REM sleep without eye movements is depicted in Figure $1 A$, and theta oscillations are evident at more anterior regions. As shown by the BOSC detection analysis on this segment of the last 5 min EEG preceding awakening, theta oscillations dominate this segment (Fig. 1B).

Similarly, Figure 2 shows a typical segment of stage 2 preceding awakening $(A)$, dominated by oscillations in the range of the delta, alpha, and sigma frequency $(B)$.

\section{REM sleep}

The typical EEG spectrum of REM sleep is depicted in Figure 3, which reports EEG power values $(0.50-24.75 \mathrm{~Hz})$ of REC and NREC groups, obtained by collapsing power values across all the derivations. An inspection of the figure suggests that EEG activity of the two groups mostly overlaps, with a notable exception for a range of frequencies encompassing upper delta and lower theta activity (from 3.25 to $6.75 \mathrm{~Hz}$ ).

The topographic distribution of EEG power, illustrated on the left side of Figure 4, depicts EEG power maps for each frequency band, scaled between the maximum and minimum values, in the REC and NREC groups; maps of statistical differences between groups are also reported on the right side of Figure 4 . The data of maxima and minima exhibited the typical features of power spectra during REM sleep (Marzano et al., 2010). The delta and theta bands exhibit a fronto-central midline predominance of power and minimum values over the temporal regions, whereas the highest values of the alpha band are seen at the parietal-occipital midline areas.

\section{REM sleep}

\section{REC}
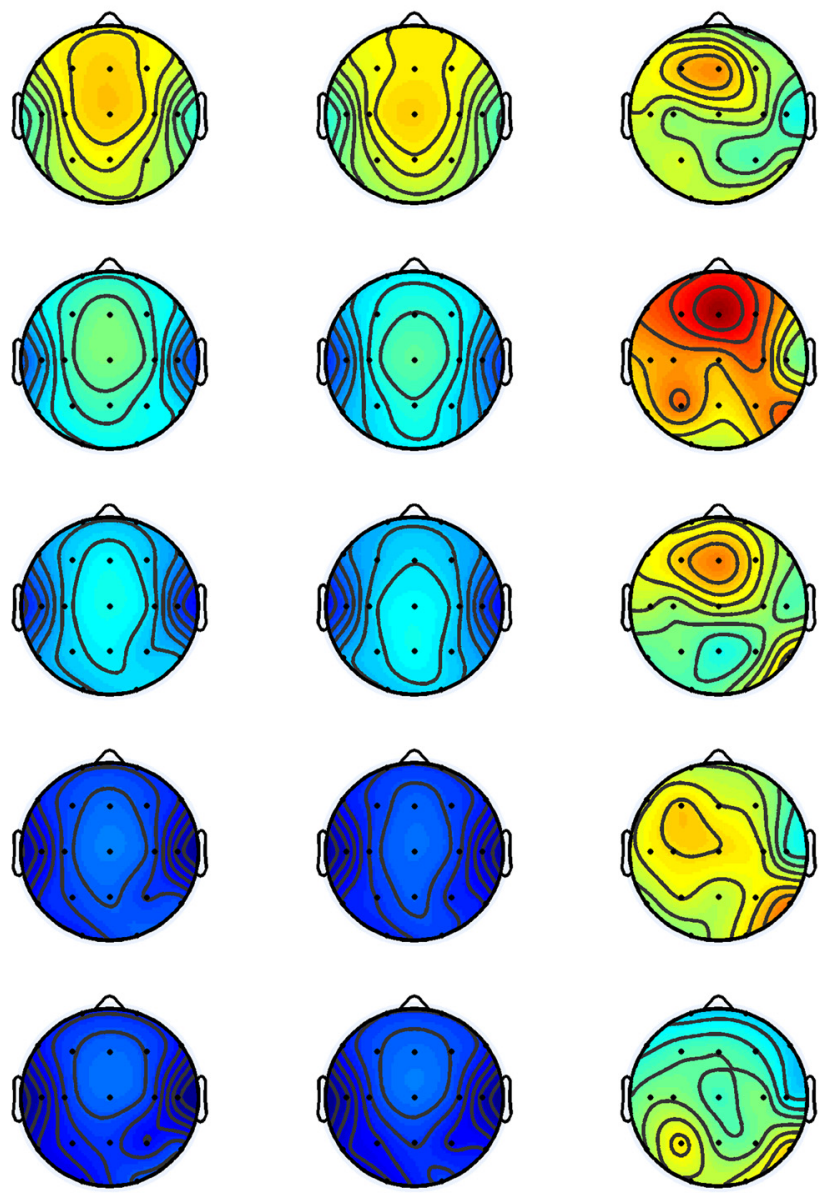

REC
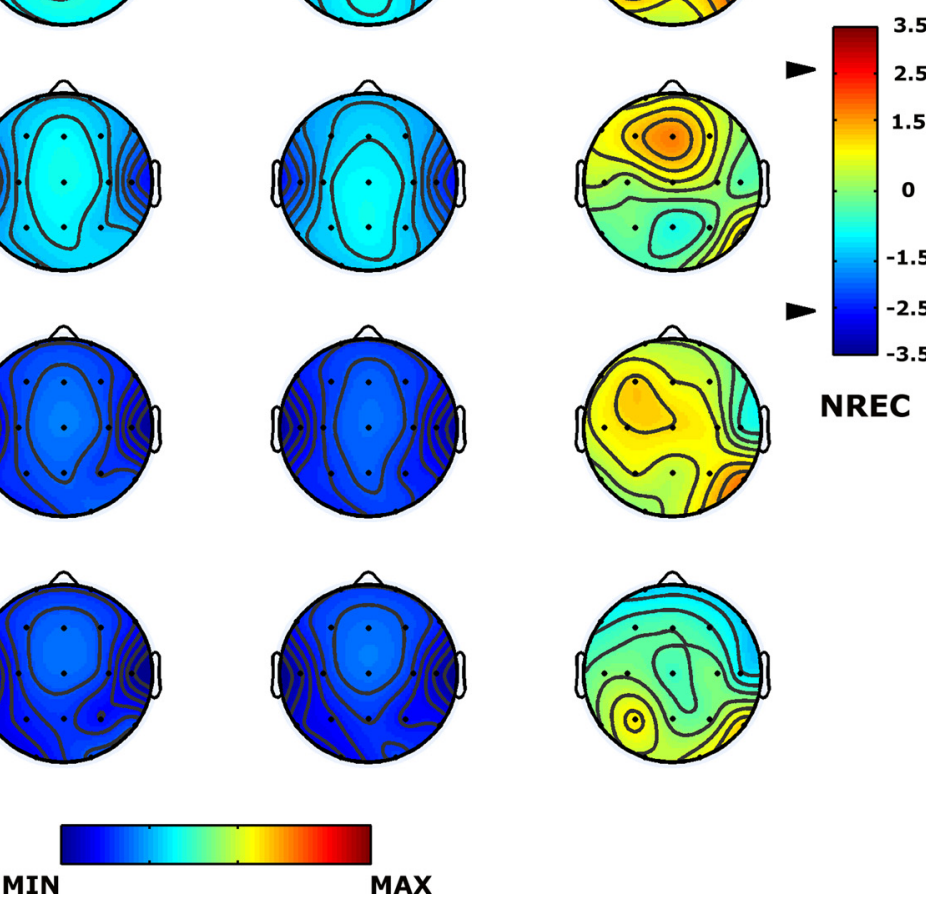

Figure 4. From the left, the first two columns show the topographic distribution of absolute EEG power during REM sleep in the REC group and in the NREC group. The maps were scaled between minimal ( $\mathrm{min}$ ) and maximal ( $m a x$ ) power values in the REC and NREC groups. The first column on the right shows topographic statistical EEG power differences (assessed by unpaired $t$ tests) between the REC and NREC groups. Values are expressed in terms of $t$ values: positive $t$ values indicate a prevalence of the REC over the NREC group, and vice versa. The two-tailed level of significance $(p \leq 0.00738)$ corresponds to $t \geq 2.60$. Average values are normalized by total power, color coded, plotted at the corresponding position on the planar projection of the scalp surface, and interpolated (biharmonic spline) between electrodes. The maps are based on the 19 unipolar EEG derivations of the international $10-20$ system with averaged mastoid reference. Maps are plotted for the following EEG bands: delta $(0.50-4.75 \mathrm{~Hz})$, theta $(5.00-7.75 \mathrm{~Hz})$, alpha $(8.00-11.75 \mathrm{~Hz})$, sigma $(12.00-15.75 \mathrm{~Hz})$, and beta $(16.00-24.75 \mathrm{~Hz})$.

A $2 \times 19 \times 5$ mixed-model ANOVA, condition (REC vs NREC) $\times$ electrode $\times$ band (delta, theta, alpha, sigma, beta), pointed to a significant condition $\times$ electrode $\times$ band interaction $\left(f_{(1,18)}=1.50 ; p<0.005\right)$. This interaction suggests that the magnitude of the differences between REC versus NREC changes as a function of both EEG bands and electrodes. In fact, the topographic distribution of statistical comparisons ( $t$ values), illustrated in the right side of Figure 4, allows assessing for which electrode and band the two groups significantly differ. These statistical maps point to a significant difference only for the theta power: REC showed higher power values than NREC subjects in the corresponding Fz scalp location $\left(t_{(28)}=3.28\right.$; two-tailed $p=0.0028$ ). 


\section{Stage 2 NREM}

The typical EEG spectrum of NREM sleep is depicted in Figure 5, which reports EEG power values $(0.50-24.75 \mathrm{~Hz})$ of the REC and NREC groups, obtained by collapsing EEG power across all derivations. An inspection of the Figure 5 suggests that EEG activity of the two groups diverges for a range of frequencies mostly encompassing the alpha and sigma bands.

Again, the topographic distributions of EEG power, illustrated in Figure 6, show the comparison of regional differences between the two groups. Figure 6 depicts EEG power maps of REC and NREC groups for each frequency band, scaled between the maximum and minimum values, and the statistical maps of the between-group differences. The topographic distributions point to stable patterns within different frequency bands. Data of maxima and minima exhibited the typical features of power spectra during NREM sleep, with a fronto-central midline predominance of delta, theta, and alpha bands and minimum values over the temporal regions [consistently with previous observations (Finelli et al., 2001; Marzano et al., 2010)], whereas the sigma band confirms centro-parietal maxima (De Gennaro and Ferrara, 2003). These regional patterns are roughly maintained in the REC and NREC groups.

A $2 \times 19 \times 5$ mixed-model ANOVA, condition (REC vs NREC) $\times$ electrode $\times$ band (delta, theta, alpha, sigma, beta), did not show a significant condition $\times$ electrode $\times$ band interaction $\left(f_{(1,18)}=0.32 ; p=0.99\right)$. The inspection of the left side of Figure 6 suggests a high similarity of the whole EEG maps of the alpha and sigma bands, and a comparable right temporo-parietal decrease associated with dream recalling across theta, alpha, and sigma bands. Likely, these regional patterns across some frequency bands had precluded the significance of the higher-order interaction, and we then performed a separate $2 \times 19$, condition $\times$ electrode ANOVA for each frequency band. The crucial condition $\times$ electrode interaction was not significant for the delta, theta, and beta bands (delta: $F_{(1,18)}=0.31, p=0.99$; theta: $F_{(1,18)}=0.59, p=0.91$; beta: $\left.F_{(1,18)}=0.71, p=0.80\right)$; approached to significance for the sigma band $\left(f_{(1,18)}=1.37 ; p=0.14\right)$; and was significant for the alpha band $\left(f_{(1,18)}=1.97 ; p=0.009\right)$. This means that the statistical maps on the right side of Figure 6 point to a significant difference only for the alpha power: REC showed lower power values than NREC subjects in the corresponding T4 scalp location $\left(t_{(28)}=-3.48\right.$; two-tailed $\left.p=0.001\right)$. A similar difference, albeit not significant after the correction for multiple testing, is apparent for the sigma activity at T4 and P4 sites and for the alpha activity at the $\mathrm{P} 4$ site (in all cases, $p=0.01$ ).

To summarize, the patterns of statistical comparisons for REM and stage 2 sleep showed that REC and NREC groups can be discriminated on the basis of the theta activity at Fz in REM sleep and the alpha activity at T4 in stage 2 NREM sleep.

\section{The increase in theta power in REM sleep and the decrease in alpha power in stage 2 also predict the number of recalled dreams}

To further support the hypothesis of a direct link between EEG activity preceding awakening and presence/absence of dream recall, we examined also whether the number of recalled dreams is predicted by the theta power after awakening from REM sleep and by the alpha power after awakening from stage 2 of NREM sleep, respectively.

Significance of these Spearman correlation coefficients was evaluated against the null hypothesis of $\rho=0$ in the general population. The alpha level for the 19 correlations in REM and

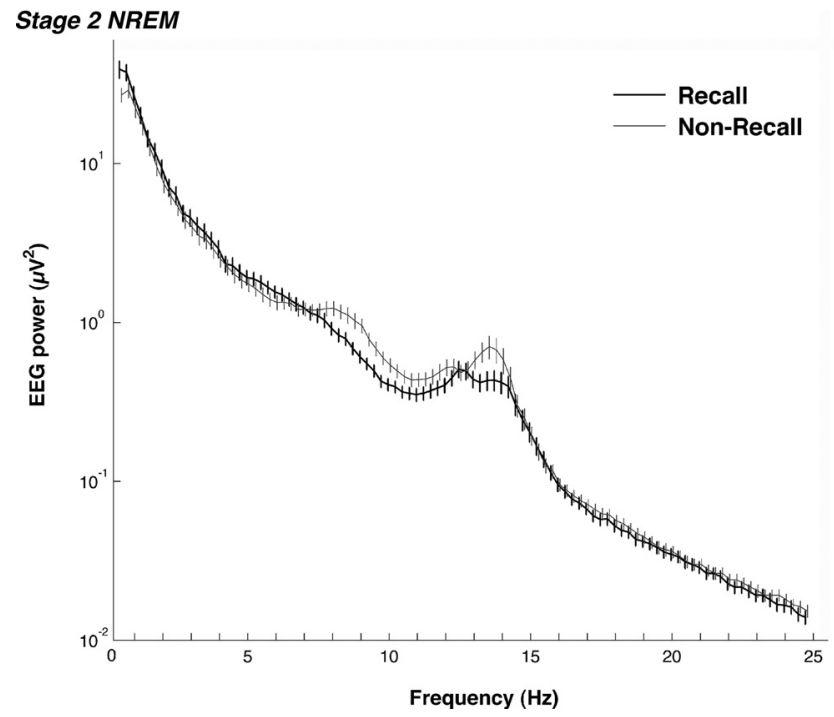

Figure 5. EEG power spectra (mean over all 19 scalp locations, obtained by collapsing EEG power across all the derivations) of EEG activity during stage 2 NREM sleep in the REC (black line) and NREC (gray line) groups. Mean absolute values (expressed in logarithmic scale) are plotted in the frequency range from 0.50 to $24.75 \mathrm{~Hz}$ for $0.25 \mathrm{~Hz}$ bins. SEs refer to interlocation variability.

the 19 ones in NREM sleep was, respectively, adjusted to 0.007 $(\rho \geq 0.47)$ and to $0.004(\rho \geq 0.46)$.

Figure 7 illustrates topographically (i.e., across all electrodes) these correlations. The number of dreams recalled after morning awakening was associated positively with the amount of theta activity in REM sleep (Fig. 7, top) and negatively with alpha activity in stage 2 (Fig. 7, bottom). Significant correlations strictly overlapped the results of between-group analyses: the positive relationship with theta activity in REM was significant only at the Fz site $(\rho=0.47 ; p=0.007)$, whereas the negative relationship with alpha activity in stage 2 was significant only at the T4 site $(\rho=-0.49 ; p=0.004)$.

\section{Oscillatory or "background" EEG activity?}

Since the FFT does not allow the discrimination between background EEG activity and an oscillatory (rhythmic) activity, we applied the BOSC detection method to the 5 min EEG segments of REM sleep or stage 2 sleep preceding the final awakening. On the basis of the above reported results, this analysis was performed on the $\mathrm{Fz}$ recordings during REM sleep and on the T4 recordings during stage 2 sleep.

BOSC analysis clearly showed that EEG activity of the preawakening segments of REM sleep contains specific oscillatory activities, mainly theta and beta (Fig. 8). Theta oscillations predominated in this 5 min of REM sleep EEG, with a mean peak of $P_{\text {episode }}$ at $6.5 \mathrm{~Hz}$ and an averaged $26 \%$ of time elapsed in this oscillatory activity. The individual maximal $P_{\text {episode }}$ values of these oscillations range from 4.9 to $7.5 \mathrm{~Hz}$. In corresponding frequencies, theta oscillations are present in the 5 min EEG segment for $9.5-40.9 \%$ of the time.

During stage 2 sleep, preawakening EEG segments mainly contained three oscillatory activities, delta, alpha, and sigma (Fig. 9), with alpha and sigma oscillations essentially predominant. Alpha oscillations show a mean peak of $P_{\text {episode }}$ at $8.5 \mathrm{~Hz}$ and an averaged $23 \%$ of time in this oscillatory activity. The individual maximal $P_{\text {episode }}$ values of these oscillations range from 7.5 to 11.3 $\mathrm{Hz}$. In corresponding frequencies, alpha oscillations are present 
in the individual 5 min EEG segments for $6.8-55.9 \%$ of the time. Sigma oscillations exhibit a mean peak at $13.0 \mathrm{~Hz}$ and an averaged $22 \%$ of time in this oscillatory activity. The individual maximal $P_{\text {episode }}$ values range from 12.1 to $14.9 \mathrm{~Hz}$. In corresponding frequencies, sigma oscillations are present in the individual $5 \mathrm{~min}$ EEG segments for $6.2-33.6 \%$ of the time.

\section{Trait-like differences or}

\section{state-like differences?}

To address the question of whether the predictive relationships between theta oscillations in REM sleep, alpha oscillations in stage 2 sleep, and dream recall apply only to the 5 min EEG segments before awakening or represent stable patterns over the night, we also examined EEG segments selected around the midpoint of the second, third, and fourth episodes of REM sleep and stage 2 NREM (see Materials and Methods).

The topographic distributions of statistical comparisons ( $t$ values) across sleep cycles regarding theta power in REM sleep and alpha power in stage 2 sleep are reported in Figure 10. No difference was significant after the correction for multiple comparisons. However, it seems worth pointing out that theta power was higher in REC compared with NREC subjects during REM sleep at $\mathrm{Fz}\left(t_{(20)}=\right.$ 3.01; two-tailed $p=0.007)$, T6 $\left(t_{(20)}=\right.$ 3.30; two-tailed $p=0.004)$, and $\mathrm{O} 2$ $\left(t_{(20)}=3.27\right.$; two-tailed $\left.p=0.004\right)$ in the fourth sleep episode, whereas no statistical comparison during stage 2 was close to significance.

According to the specific aims of these analyses, we also calculated the effect sizes (Cohen's d coefficients) for the REC versus NREC comparisons at Fz in REM sleep and at T4 in stage 2 sleep. These coefficients showed a relative increase of the differences between REC versus NREC in the EEG segment preceding the final awakening compared with the other sleep episodes (Cohen's d coefficients for REM sleep: second sleep episode, 0.79 ; third sleep episode, 0.61 ; fourth sleep episode, 1.41 ; Cohen's d coefficients for stage 2 sleep: second sleep episode, 0.86; third sleep episode, 0.89 ; fourth sleep episode, 1.09).

\section{Discussion}

Our results provide a clear electrophysiological evidence that stage-specific cortical brain oscillations in the $5 \mathrm{~min}$ of sleep before morning awakening are predictive of a successful dream recall. A higher frontal theta activity predicts successful dream recall after awakening from REM sleep, as well as a lower alpha oscillatory activity of the right temporal area after awakening from stage 2 NREM sleep. Moreover, the significant correlations with the estimated number of recalled dreams confirm the pre-

\section{Stage 2 NREM}

REC
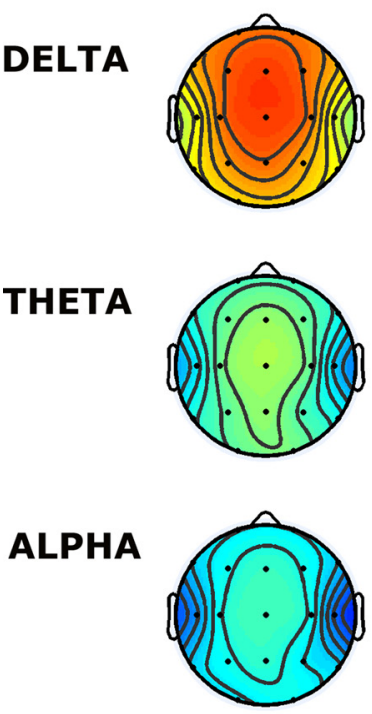

SIGMA
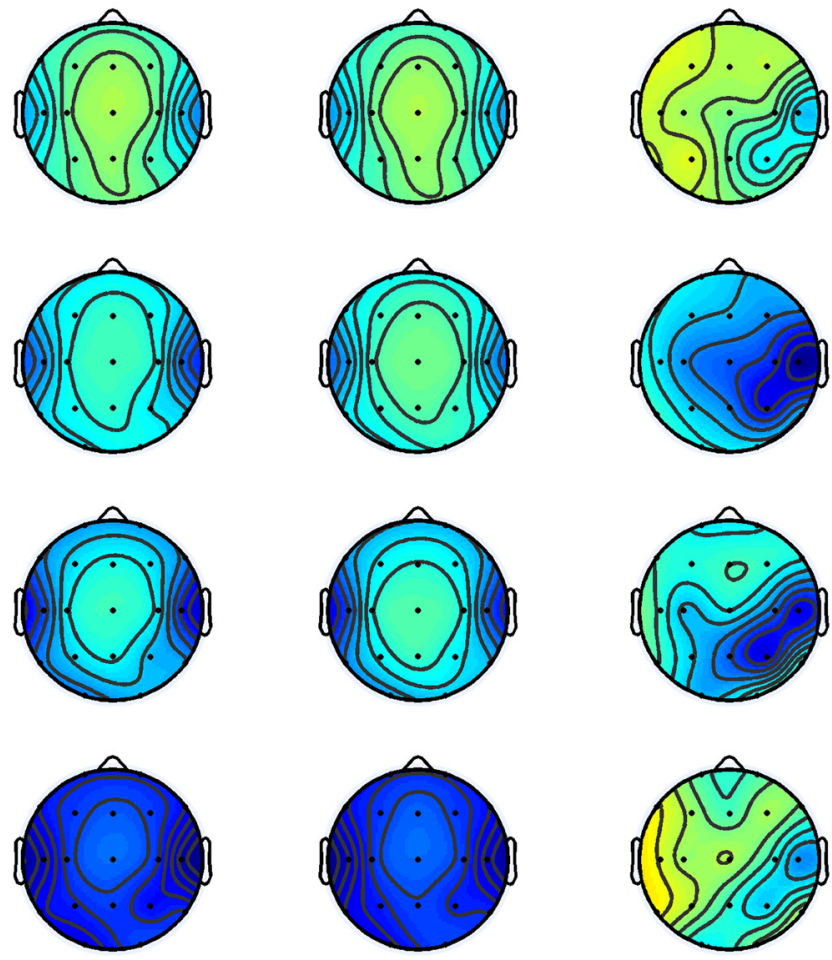

REC

Figure 6. From the left, the first two columns show the topographic distribution of absolute EEG power during stage 2 NREM sleep in the REC and NREC groups. The maps were scaled between minimal ( $\mathrm{min}$ ) and maximal ( $\mathrm{max}$ ) power values in the REC and NREC groups. The first column on the right shows topographic statistical EEG power differences (assessed by unpaired $t$ tests) between the REC and NREC groups. Values are expressed in terms of $t$ values: positive $t$ values indicate a prevalence of the REC ove the NREC group, and vice versa. The two-tailed level of significance $(p \leq 0.00447)$ corresponds to at $t \geq 2.78$. Average values are normalized by total power, color coded, plotted at the corresponding position on the planar projection of the scalp surface, and interpolated (biharmonic spline) between electrodes. The maps are based on the 19 unipolar EEG derivations of the internationa $10-20$ system with averaged mastoid reference. Maps are plotted for the following EEG bands: delta $(0.50-4.75 \mathrm{~Hz})$, theta $(5.00-7.75 \mathrm{~Hz})$, alpha $(8.00-11.75 \mathrm{~Hz})$, sigma $(12.00-15.75 \mathrm{~Hz})$, and beta $(16.00-24.75 \mathrm{~Hz})$. dictive power of frontal theta activity in REM sleep and of right temporal alpha activity in NREM sleep for dream recall.

Although the identification of oscillatory activity in quasistationary EEG traces (i.e., in a relatively short interval, in which the parameters of interest vary minimally) is still an open question, theta and alpha power values extracted by the FFT, respectively, in REM and stage 2 NREM sleep actually appear to subtend an oscillatory activity. As a matter of fact, the BOSC detection method confirmed that a large part of EEG segments of REM sleep and stage 2 before morning awakening contains theta and alpha oscillations, respectively.

Together, the changes of cortical oscillatory activity during sleep localized over the frontal and temporo-occipital regions 


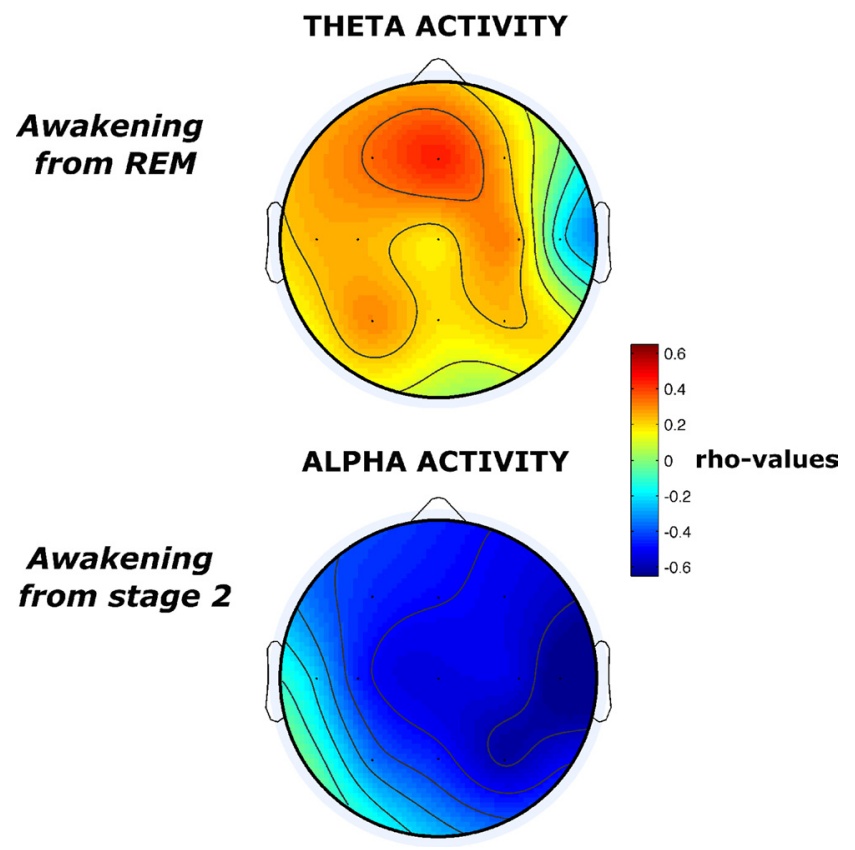

Figure 7. The topographic distribution of correlation values (rho values) between the actual number of dreams recalled after morning awakenings with the amount of theta activity in REM sleep (top map) and with alpha activity in stage 2 (bottom map). Values are expressed in terms of rho values: positive rho values indicate the presence of a positive correlation, and vice versa. The alpha level for REM and NREM sleep was, respectively, adjusted to $0.007(\rho \geq 0.47)$ and to 0.004 ( $\rho \geq 0.46$ ). Average values were normalized by total power, color coded, plotted at the corresponding position on the planar projection of the scalp surface, and interpolated (biharmonic spline) between electrodes. The maps are based on the 19 unipolar EEG derivations of the international $10-20$ system with averaged mastoid reference.

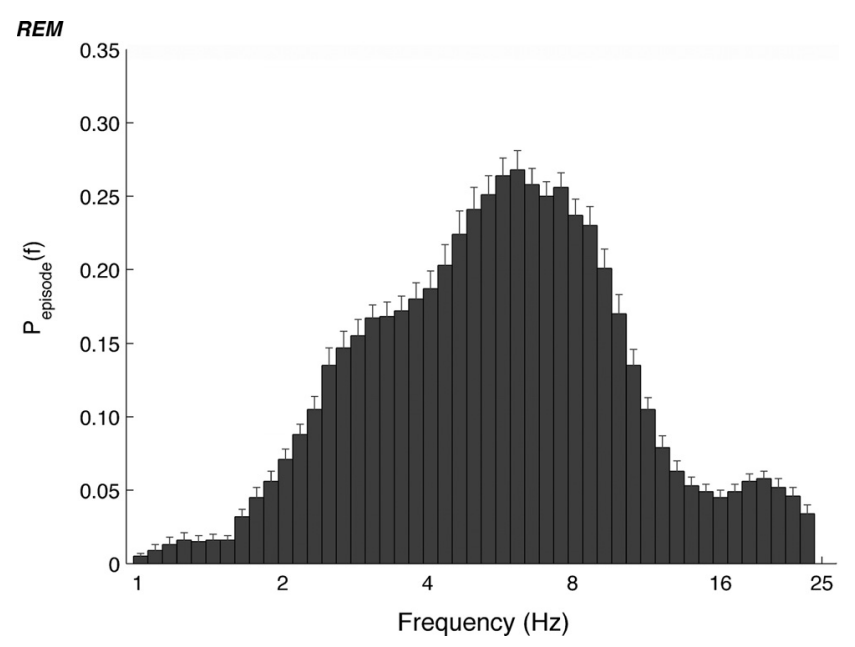

Figure 8. Mean proportion of time $\left[P_{\text {episode }}(f)\right]$ of EEG activity during REM sleep during which oscillations were detected at each frequency in the $0.50-25.00 \mathrm{~Hz}$ frequency range. Individual oscillations detected across all frequencies by the BOSC method have been averaged across all subjects, and error bars denote SEM $P_{\text {episodes }}(f)$. Units of frequency are in hertz and are plotted in five logarithmically spaced frequency values. See Materials and Methods for more details on the BOSC method.

appear indicative of the involvement also in dreaming of those regions, which control successful memory encoding in waking. In more general terms, our results suggest that the neurophysiological mechanisms underlying the encoding and recall of episodic memories remain the same across different states of consciousness.

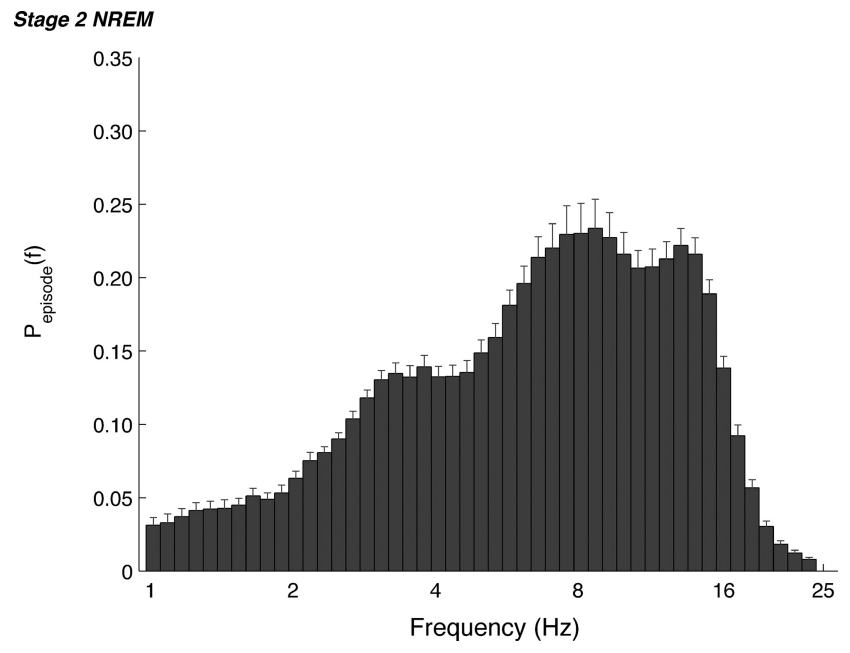

Figure 9. Mean proportion of time $\left[P_{\text {episode }}(f)\right]$ of EEG activity during stage 2 sleep during which oscillations were detected by the BOSC method at each frequency in the $0.25-25.00 \mathrm{~Hz}$ frequency range, as in Figure 8.

It is of note that the cortical regions whose oscillatory activity predicts dream recall are reminiscent of those involved in the global cessation of dreaming after brain injuries. The largest investigation on 361 brain-damaged patients (Solms, 1997, 2000) showed that global cessation of dreaming follows a lesion of two different cortical regions: (1) the temporoparieto-occipital junction, more often unilaterally than bilaterally (Solms, 2000); and (2) white matter tracts surrounding the frontal horns of the lateral ventricles, underlying ventromedial prefrontal cortex, more often bilaterally than unilaterally (Solms, 1997).

\section{The continuity hypothesis}

The present findings confirm the basic assumption that the neurophysiological mechanisms of encoding and recall of episodic memories are homogeneous across wake and sleep, but also complement the so-called "continuity hypothesis" of dreaming. This hypothesis states that (1) qualitative and quantitative aspects of dreams largely reflect waking-life experience (Domhoff, 2003; Schredl, 2003; Schredl, 2009) and (2) the likelihood of specific experiences, thoughts, and feelings of the waking life to be incorporated into subsequent dreams is modulated by several factors [such as emotional involvement, type of waking-life experience, personality traits, and time of the night (Schredl, 2003, 2009)]. Beyond this essentially psychological perspective, the hypothesis has been supported by a recent neuroimaging study that showed direct relationships between volumetric and ultrastructural measures of the hippocampus-amygdala complex and specific qualitative (mainly, emotions and bizarreness) features of dreaming (De Gennaro et al., 2010). In fact, the amygdala, involved during wakefulness in both control of the encoding and retrieval of emotional memories and the physical expression of emotions (Misane et al., 2005), also seems involved in the access and processing of the emotional sources of dreaming (Hobson and Pace-Schott, 2002).

The current EEG results, in suggesting that the mechanisms involved in encoding and recall of episodic memories across wakefulness and sleep are the same, undoubtedly strengthen the general notion of a continuity between waking and sleep mentation. 


\section{NREM dream recall}

Our findings also allow us to further clarify the differences in dream recall after awakening from NREM and REM sleep.

In our view, the different findings for REM and NREM sleep are consistent with both the quantitative (and, probably, also qualitative) differences in dreams reported after awakening from the two states (Nielsen, 2000), and the peculiar physiology of REM sleep, whose electrophysiological and neurochemical milieu resembles that of wakefulness more than that of NREM sleep (Nir and Tononi, 2010). Positron emission tomography studies have also shown that global brain metabolism is comparable between wakefulness and REM sleep (Braun et al., 1997; Maquet, 2000).

Our finding of a decreased alpha activity in stage 2 NREM sleep substantially replicates the finding by Esposito et al. (2004), who showed that successful recall after awakening from stage 2 is associated with a lower alpha power, particularly in corresponding centro-temporo-parietal areas. Also during wakefulness, a more efficient encoding of words and faces has been shown to be associated with a lower alpha activity $(10-12 \mathrm{~Hz})$ in the right temporo-parietal cortical region (Molle et al., 2002). Interestingly, Molle et al. (2002) also found an increased frontal and temporal theta activity when comparing trials with efficient versus poor encoding. Moreover, intracranial recordings of awake humans have given further support for an association between a successful episodic memory encoding and a decreased temporo-parietal alpha activity (Sederberg et al., 2003). Complementarily, increases in alpha power have been correlated with decreased memory performance (Klimesch et al., 1997; Klimesch, 1999), and EEG-functional magnetic resonance imaging recordings have shown that alpha activity is negatively correlated with the BOLD signal in the medial prefrontal cortex, primary visual cortex, and middle temporal gyrus during memory retention (Scheeringa et al., 2009).

During NREM sleep, alpha oscillations are indicative of a cortical EEG synchronization, since it increases during postdeprivation recovery sleep (Marzano et al., 2010) and is positively correlated with delta-theta activity in the first sleep cycle (Ferrara et al., 2002). Notably, decreases of alpha activity in NREM sleep are associated with corresponding increases in REM sleep (Marzano et al., 2010). Together, these findings suggest that the decreased alpha activity in stage 2 sleep, which is likely to involve the thalamo-cortical network (Klimesch, 1999), is indicative of a lower cortical activation, which is in turn associated with a decreased likelihood of successful dream recall after awakening.

\section{Stable interindividual differences?}

According to the results obtained by analyzing three consecutive sleep cycles, the current findings do not support an interpretaion in terms of a stable interindividual EEG pattern characterizing subjects in the entire night. Actually, the pattern of topographical differences for the theta activity after REM sleep awakenings and for the alpha activity after stage 2 awakenings suggests that the predictive relationship with dream recall is a function of the proximity to the time of awakening. In other words, the closer the time of collecting 3rd sleep episode

4th sleep episode
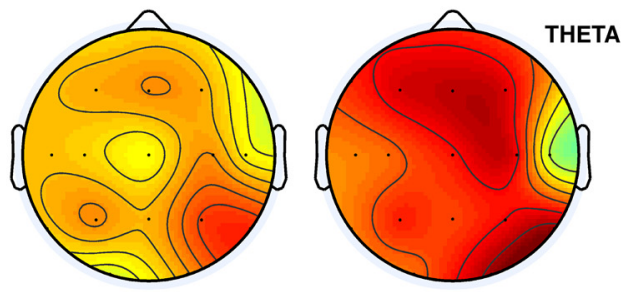

REC

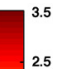

1.5
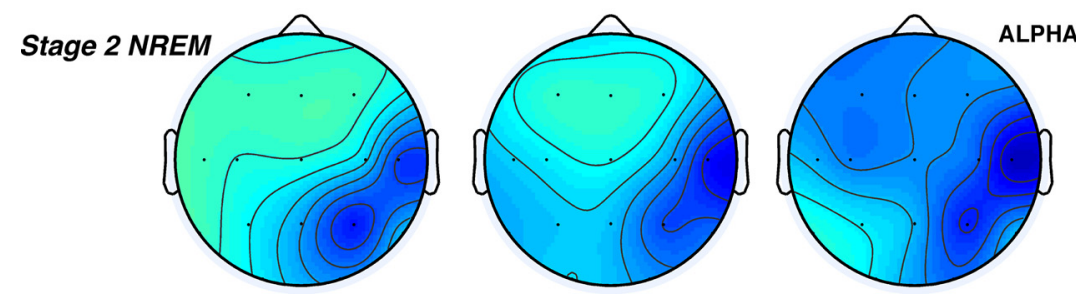

-1.5
-2.5
-3.5

NREC

Figure 10. Theta activity during REM sleep and alpha activity during stage 2 across consecutive sleep cycles. The maps report position on the planar projection of the scalp surface, and interpolated (biharmonic spline) between electrodes. The maps are

dream reports, the larger between-group differences we find. However, the current study cannot provide a definitive response to this specific question. This issue can be disentangled only by multiplenight within-subject studies, in which preawakening sleep periods followed by successful/failed dream recall could be compared and, complementarily, from multinight studies on subjects who stably recall or do not recall dreams.

\section{Concluding remarks}

The current model of episodic memory based on human studies (for review, see Nyhus and Curran, 2010) states that theta oscillations allow for top-down control from the frontal cortex to the hippocampus, modulating the encoding and retrieval of episodic memories. The theta activity is also higher during encoding than retrieval, being related more to the access of a stored code than to more general processes (Klimesch et al., 2001). On the other hand, the alpha activity seems functionally related to the activation of stored information, and both the alpha power decrease and the theta power increase are functionally independent (Khader and Rösler, 2010). Our results, in showing that success/ failure of dream recall is related to specific cortical EEG activity and oscillations during the sleep stage preceding awakening, also suggest that the intrinsic differences in the basic electrophysiology of the NREM stage 2 and REM sleep may determine which cortical EEG oscillation (i.e., frontal theta or right temporal alpha) is predictive of dream recall after awakening. In this view, the alpha oscillations likely involve the thalamo-cortical network, whereas the theta rhythm likely involves the hippocampal-cortical network, and these networks might be differently related to semantic and episodic memory (Klimesch, 1999; Nyhus and Curran, 2010). Suggestively, this dissociation resembles some differences between qualitative features of dream reports after awakenings from REM and NREM sleep (for review, see Nielsen, 2000). Also this suggestion, currently mostly speculative, may be tested by combining the intracranial recordings of hippocampal 
and prefrontal regions during sleep preceding the awakening with the postawakening collection of dream report in epileptic patients with intracranial implanted electrodes.

\section{References}

Anderson KL, Rajagovindan R, Ghacibeh GA, Meador KJ, Ding M (2010) Theta oscillations mediate interaction between prefrontal cortex and medial temporal lobe in human memory. Cereb Cortex 20:1604-1612.

Braun AR, Balkin TJ, Wesenten NJ, Carson RE, Varga M, Baldwin P, Selbie S, Belenky G, Herscovitch P (1997) Regional cerebral blood flow throughout the sleep-wake cycle. An H2(15)O PET study. Brain 120:1173-1197.

Caplan JB, Glaholt MG (2007) The roles of EEG oscillations in learning relational information. Neuroimage 38:604-616.

Caplan JB, Madsen JR, Raghavachari S, Kahana MJ (2001) Distinct patterns of brain oscillations underlie two basic parameters of human maze learning. J Neurophysiol 86:368-380.

Caplan JB, Madsen JR, Schulze-Bonhage A, Aschenbrenner-Scheibe R, Newman EL, Kahana MJ (2003) Human theta oscillations related to sensorimotor integration and spatial learning. J Neurosci 23:4726-4736.

De Gennaro L, Ferrara M (2003) Sleep spindles: an overview. Sleep Med Rev 75:423-440.

De Gennaro L, Fratello F, Marzano C, Moroni F, Curcio G, Tempesta D, Pellicciari MC, Pirulli C, Ferrara M, Rossini PM (2008) Cortical plasticity induced by transcranial magnetic stimulation during wakefulness affects electroencephalogram activity during sleep. PLoS ONE 3:e2483.

De Gennaro L, Marzano C, Moroni F, Curcio G, Ferrara M, Cipolli C (2010) Recovery sleep after sleep deprivation almost completely abolishes dream recall. Behav Brain Res 206:293-298.

Domhoff GW (2003) The scientific study of dreams: neural networks, cognitive development, and content analysis. Washington, DC: American Psychological Association.

Ekstrom AD, Caplan JB, Ho E, Shattuck K, Fried I, Kahana MJ (2005) Human hippocampal theta activity during virtual navigation. Hippocampus $15: 881-889$

Esposito MJ, Nielsen TA, Paquette T (2004) Reduced alpha power associated with the recall of mentation from stage 2 and stage REM sleep. Psychophysiology 41:288-297.

Fagioli I (2002) Mental activity during sleep. Sleep Med Rev 6:307-320.

Feinberg I, Floyd TC (1979) Systematic trends across the night in human sleep cycles. Psychophysiology 16:283-291.

Ferrara M, De Gennaro L, Curcio G, Cristiani R, Bertini M (2002) Regional differences of the temporal EEG dynamics during the first 30 minutes of human sleep. Neurosci Res 44:83-89.

Finelli LA, Borbely AA, Achermann P (2001) Functional topography of the human nonREM sleep electroencephalogram. Eur J Neurosci 13:2282-2290.

Fosse MJ, Fosse R, Hobson JA, Stickgold RJ (2003) Dreaming and episodic memory: a functional dissociation? J Cogn Neurosci 15:1-9.

Hobson JA, Pace-Schott EF (2002) The cognitive neuroscience of sleep: neuronal systems, consciousness and learning. Nat Rev Neurosci 3:679-693.

Khader PH, Rösler F (2010) EEG power changes reflect distinct mechanisms during long-term memory retrieval. Psychophysiology 48(3):362-369.

Klimesch W (1999) EEG alpha and theta oscillations reflect cognitive and memory performance: a review and analysis. Brain Res Rev 29:169-195.

Klimesch W, Doppelmayr M, Russegger H, Pachinger T (1996) Theta band power in the human scalp EEG and the encoding of new information. Neuroreport 7:1235-1240.

Klimesch W, Doppelmayr M, Schimke H, Ripper B (1997) Theta synchronization and alpha desynchronization in a memory task. Psychophysiology 34:169-176.

Klimesch W, Doppelmayr M, Stadler W, Pollhuber D, Sauseng P, Rohm D
(2001) Episodic retrieval is reflected by a process specific increase in human electroencephalographic theta activity. Neurosci Lett 302:49-52.

Maquet P (2000) Functional neuroimaging of normal human sleep by positron emission tomography. J Sleep Res 9:207-231.

Marzano C, Ferrara M, Curcio G, De Gennaro L (2010) The effects of sleep deprivation in humans: topographical EEG changes in NREM vs. REM sleep. J Sleep Res 19:260-268.

Misane I, Tovote P, Meyer M, Spiess J, Ogren SO, Stiedl O (2005) Timedependent involvement of the dorsal hippocampus in trace fear conditioning in mice. Hippocampus 15:418-426.

Molle M, Marshall L, Fehm HL, Born J (2002) EEG theta synchronization conjoined with alpha desynchronization indicate intentional encoding. Eur J Neurosci 15:923-928.

Nielsen TA (2000) A review of mentation in REM and NREM sleep: "covert" REM sleep as a possible reconciliation of two opposing models. Behav Brain Sci 23:851-866.

Nir Y, Tononi G (2010) Dreaming and the brain: from phenomenology to neurophysiology. Trends Cogn Sci 14:88-100.

Nishida M, Pearsall J, Buckner RL, Walker MP (2009) REM sleep, prefrontal theta, and the consolidation of human emotional memory. Cereb Cortex 19:1158-1166.

Nyhus E, Curran T (2010) Functional role of gamma and theta oscillations in episodic memory. Neurosci Biobehav Rev 34:1023-1035.

Osipova D, Takashima A, Oostenveld R, Fernandez G, Maris E, Jensen O (2006) Theta and gamma oscillations predict encoding and retrieval of declarative memory. J Neurosci 26:7523-7531.

Paller KA, Wagner AD (2002) Observing the transformation of experience into memory. Trends Cogn Sci 6:93-102.

Perneger TV (1998) What is wrong with Bonferroni adjustments. BMJ 136:1236-1238

Rechtschaffen A, Kales A (1968) A manual of standardized terminology, techniques and scoring system for sleep stages of human subjects. Los Angeles: UCLA Brain Information Service.

Rutishauser U, Ross IB, Mamelak AN, Schuman EM (2010) Human memory strength is predicted by theta-frequency phase-locking of single neurons. Nature 464:903-907.

Sankoh AJ, Huque MF, Dubey SD (1997) Some comments on frequently used multiple endpoint adjustments methods in clinical trials. Stat Med 16:2529-2542.

Scheeringa R, Petersson KM, Oostenveld R, Norris DG, Hagoort P, Bastiaansen MC (2009) Trial-by-trial coupling between EEG and BOLD identifies networks related to alpha and theta EEG power increases during working memory maintenance. Neuroimage 44:1224-1238.

Schredl M (2003) Continuity between waking and dreaming: a proposal for a mathematicalmodel. Sleep Hypnosis 5:26-40.

Schredl M (2009) Dreams in patients with sleep disorders. Sleep Med Rev 13:215-221.

Sederberg PB, Kahana MJ, Howard MW, Donner EJ, Madsen JR (2003) Theta and gamma oscillations during encoding predict subsequent recall. J Neurosci 23:10809-10814.

Solms M (1997) The neuropsychology of dreaming: a clinico-anatomical study. Mawah, NJ; Lawrence Erlbaum.

Solms M (2000) Dreaming and REM sleep are controlled by different brain mechanisms. Behav Brain Sci 23:843-850.

van Vugt MK, Sederberg PB, Kahana MJ (2007) Comparison of spectral analysis methods for characterizing brain oscillations. J Neurosci Methods 162:49-63.

Weiss S, Rappelsberger P (2000) Long-range EEG synchronization during word encoding correlates with successful memory performance. Cogn Brain Res 9:299-312.

Whitten TA, Hughes AM, Dickson CT, Caplan JB (2010) A better oscillation detection method robustly extracts EEG rhythms across brain state changes: the human alpha rhythm as a test case. Neuroimage 54:860 -874. 